\title{
Reduced angiotensinogen expression attenuates renal interstitial fibrosis in obstructive nephropathy in mice
}

\author{
Robert J. Fern, ${ }^{1}$ Christine M. Yesko, ${ }^{1}$ Barbara A. Thornhill,,${ }^{1}$ Hyung-Suk Kim, ${ }^{2}$ \\ Oliver Smithies, ${ }^{2}$ and Robert L. Chevalier ${ }^{1}$ \\ ${ }^{1}$ Department of Pediatrics, University of Virginia School of Medicine, Charlottesville, Virginia 22908, USA
${ }^{2}$ Department of Pathology, University of North Carolina, Chapel Hill, North Carolina 27599, USA \\ Address correspondence to: Robert L. Chevalier, Department of Pediatrics, Box 386, University of Virginia Health Sciences Center, \\ Charlottesville, Virginia 22908, USA. Phone: (804) 924-5093; Fax: (804) 982-3561; E-mail: rlc2m@virginia.edu
}

Received for publication June 9, 1998, and accepted in revised form November 10, 1998.

\begin{abstract}
A novel approach was employed to assess the contribution of the renin-angiotensin system (RAS) to obstructive nephropathy in neonatal mice having zero to four functional copies of the angiotensinogen gene (Agt). Two-day-old mice underwent unilateral ureteral obstruction (UUO) or sham operation; 28 days later, renal interstitial fibrosis and tubular atrophy were quantitated. In all Agt genotypes, UUO reduced ipsilateral renal mass and increased that of the opposite kidney. Renal interstitial collagen increased after UUO linearly with Agt expression, from a fractional area of $25 \%$ in zero-copy mice to $54 \%$ in two-copy mice. Renal expression of transforming growth factor- $\beta 1$ was increased by ipsilateral UUO in mice expressing Agt, but not in zero-copy mice. However, the prevalence of atrophic tubules due to UUO did not vary with Agt expression. Blood pressure was not different in all groups, except for a reduction in sham zero-copy mice. We conclude that a functional RAS is not necessary for compensatory renal growth. This study demonstrates conclusively that angiotensin regulates at least $50 \%$ of the renal interstitial fibrotic response in obstructive nephropathy, an effect independent of systemic hemodynamic changes. Angiotensininduced fibrosis likely is a mechanism common to the progression of many forms of renal disease.
\end{abstract}

J. Clin. Invest. 103:39-46 (1999).

\section{Introduction}

The renin-angiotensin system (RAS) may serve pathogenic as well as compensatory salutary roles in renal disease and may damage other organ systems, including blood vessels, heart, lungs, and liver (1). Pharmacological reduction of RAS activity improves renal pathology and function in numerous clinical and experimental conditions (reviewed in refs. 2 and 3). Conversely, chronic infusion of angiotensin (Ang) II produces marked glomerular and tubulointerstitial injury in the kidney (4-6).

A component of the renal disease-promoting action of Ang II has been attributed to the hormone's modulation of systemic and renal hemodynamics, particularly the injurious elevation of glomerular capillary pressure (7). Moreover, Ang II regulates the production of intermediary growth factors and cytokines involved in the progression of renal disease, including transforming growth factor- $\beta$ (TGF- $\beta$ ) (3), platelet-derived growth factor (PDGF) (4), endothelin (8), epidermal growth factor $(9,10)$, and tumor necrosis factor- $\alpha(11)$.

To advance the understanding of the quantitative contribution of the entire RAS to the progression of renal disease, the present study relies on a gene-targeting model in which angiotensinogen (AGT), and thus angiotensin, is varied from none to supraphysiological by altering the number of copies of Agt. Mice with zero, one, two, three, or four Agt copies at the same locus exhibit graded differences in steady-state plasma AGT levels $(12,13)$. Such a system affords an examination of the effect of multiple levels of RAS activation without requiring exogenous agonist or antagonist treatment. In fact, this strategy has demonstrated a causal relationship between Agt expression and blood pressure changes (13).

Agt targeting is applied here to chronic obstructive kidney disease secondary to complete unilateral ureteral obstruction (UUO) in the neonatal period. In this study, mice with zero to four Agt copies were subjected to UUO. Obstructive nephropathy, among the most frequent causes of end-stage renal disease in children (14), manifests itself as a tubulointerstitial disease, with progressive tubular dilatation and atrophy, as well as an expansive interstitial fibrosis (15). Upregulation of the renal RAS attends both early postnatal development and the progression of hydronephrosis after UUO. Compared with mature animals, neonates exhibit increased renal renin expression and a more extensive renal microvascular distribution of renin (16); renal Ang I and Ang II are also elevated (17). Neonatal UUO further augments renal renin expression and content and increases the number of renin-secreting cells in the renal arterioles $(18,19)$. Renal Agt expression, angiotensinconverting enzyme (ACE) activity, Ang II content, and angiotensin type $1\left(\mathrm{AT}_{1}\right)$ receptor expression and binding similarly are stimulated in the chronically obstructed kidney $(20,21)$. Moreover, ACE inhibition or $\mathrm{AT}_{1}$ receptor antagonism mitigates renal tubular and interstitial injury due to $\operatorname{UUO}(9,22)$. Therefore, UUO in developing animals with genetically determined variations in Agt expression was investigated to evaluate the quantitative impact of changes in RAS activity on renal interstitial fibrosis and tubular atrophy, hallmarks of chronic obstructive nephropathy. 

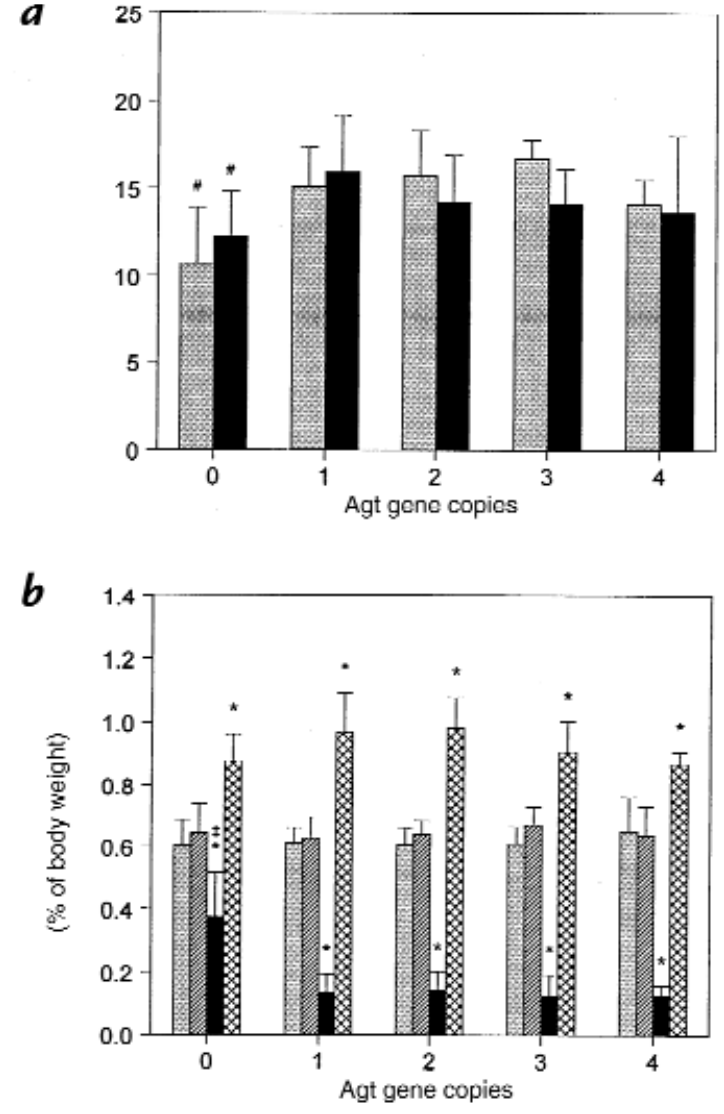

\section{Figure 1}

Effect of angiotensinogen gene (Agt) expression and UUO on body and kidney weights. (a) Body weight (in grams) was measured 28 days after complete UUO (black bars) or sham operation (gray bars) in 2-day-old mice with zero to four functional Agt copies (genotypes 0/0, 1/0, 1/1, $2 / 1$, and $2 / 2$, respectively). (b) Sham left (gray bars), sham right (hatched bars), obstructed (black bars), and intact opposite (cross-hatched bars) kidneys from the same mice in a were weighed and normalized to body weight. In sham wild-type (1/1) mice, the left renal mass ( $89 \pm 14 \mathrm{mg})$ was $0.59 \pm 0.05 \%$ of body weight. Data represent mean \pm SD. For each mean value, $n=6-10,30-48,13-19,4-6$, and 3 mice with zero, one, two, three, or four Agt copies, respectively. ${ }^{\#} P<0.05$ vs. one-copy (Agt $1 / 0$ ) mice. ${ }^{*} P<0.05$ vs. sham. ${ }^{\ddagger} P<0.05$ vs. other Agt genotypes. UUO, unilateral ureteral obstruction.

\section{Methods}

Mice with variable numbers of functional Agt genes. Agt disruption by conventional gene targeting (13) and Agt duplication by gaprepair gene targeting (12) were employed to generate mice with zero to four functional copies of Agt while preserving the gene's chromosomal location and regulatory elements. Agt targeting was performed in mouse strain 129 , which was cross-bred with mouse strain $\mathrm{C} 57 \mathrm{BL} / 6 \mathrm{~J}$ to generate $\mathrm{F}_{1}$ animals. Two separate sets of mice were maintained. Intercrossing one-copy $(A g t 1 / 0)$ mice yielded offspring with zero (0/0), one (1/0), or two (1/1) Agt copies; threecopy $($ Agt $2 / 1)$ mice were mated to produce two- $(1 / 1)$, three- $(2 / 1)$, or four-copy (2/2) progeny. Data from wild-type, or two-copy (Agt $1 / 1)$, mice from each set did not differ significantly.

Surgical procedure and tissue preparation. Forty-eight hours after birth, male and female $\mathrm{F}_{2}$ mice with varying Agt genotypes were subjected to complete left ureteral obstruction $(n=100)$ or a sham operation $(n=102)$ under general anesthesia with halothane and oxygen. With the aid of a Wild M3Z stereomi- croscope (Leica, Heerbrugg, Switzerland), the distal ureter was exposed through a longitudinal 5-mm left abdominal incision and ligated twice with 6-0 silk suture (but not transected between ligatures). In sham operations, the ureter was exposed and repositioned without further manipulation. The incision was closed in a single layer and coated with collodion. After recovery in a warmed environment, neonatal mice were returned to their mothers. Mice were weaned at 19 days of age and maintained on a standard rat chow (7012; Harlan Teklad Laboratory, Winfield, Iowa, USA) and water ad libitum until sacrifice 28 days after surgery ( 30 days of age). Kidneys were decapsulated in vivo, transferred to ice-cold saline, bisected coronally, blotted dry on gauze, and weighed. Each renal hemisection was fixed in Bouin's solution (Sigma Chemical Co., St. Louis, Missouri, USA) for $2 \mathrm{~h}$ before transfer to $70 \%$ ethanol. Bouin'sfixed renal tissue was dehydrated through an ethanol series and xylene, embedded in paraffin, and sectioned $(3 \mu \mathrm{m})$ on an RM 2155 microtome (Leica).

Genotype determination. Genomic DNA was extracted from mouse tail biopsies (collected at weaning) using phenol/chloroform extraction after proteinase $\mathrm{K}(0.5 \mathrm{mg} / \mathrm{ml})$ digestion. $A g t$ copy number was determined by PCR as described previously (13). Samples from progeny of Agt 1/0 crosses were tested in parallel reactions for the presence of disrupted ("knockout") and wild-type Agt. Primer pair 1 (5'-TGC ACG GGT TCT GAG GAT CCA-3' and 5'-TAA AGC GCA TGC TCC AGA CT-3') yields a $1.2-\mathrm{kb}$ fragment diagnostic of Agt disruption. Amplification of a 780-bp product by primer pair $2\left(5^{\prime}\right.$-GTA TAC ATC CAC CCC TTC CA-3' and 5'-GGA AGT GAA CGT AGG TGT TGA A-3') independently confirms the untargeted gene to distinguish Agt 0/0 and 1/0 mice. Agt duplication in offspring of Agt 2/1 interbreedings was assessed by primer pair 3 (5'-ACC ACT CAC GGA AGC TGC AT-3' and 5'-ACG CGT CAC CTT AAT ATG CG-3'), which produces a diagnostic 2.0$\mathrm{kb}$ fragment. Three- $(2 / 1)$ and four- $(2 / 2)$ copy mice were distinguished by the D8MIT56 sequence polymorphism (13), closely linked to the Agt locus and differing in length between mouse strains 129 (182 bp) and C57BL/6J (162 bp) (primer pair 4: 5'-ACA CTC AGA GAC CAT GAG TAC ACC-3' and 5'GAG TTC ACT ACC CAC AAG TCT CC-3'). In the absence of crossovers in this study, the strain C57BL/6J allele always furnishes the untargeted (wild-type) Agt, whereas the Agt duplication is derived from strain 129.

Histomorphometric analysis. Midcoronal renal cross-sections (including a papilla) were deparaffinized in xylene and rehydrated through graded ethanol and then stained with Masson's trichrome or periodic acid-Schiff (PAS) to evaluate interstitial collagen deposition and tubular atrophy, respectively. Histomorphometric analysis was performed by investigators (R.J. Fern, C.M. Yesko) blinded to Agt genotype. For Masson's trichrome staining, after mordanting overnight in Bouin's solution, kidney sections were treated sequentially with Gill's No. 2 hematoxylin $\times 2$ min, Biebrich scarlet-acid fuchsin $\times 5 \mathrm{~s}$, phosphotungstic acid/phosphomolybdic acid $\times 5 \mathrm{~min}$, and aniline blue $\times 15 \mathrm{~min}$. Tissue was destained in $1 \%$ acetic acid $\times 2 \mathrm{~min}$, dehydrated through graded ethanol to xylene, and mounted for examination by light microscopy. To quantitate renal interstitial collagen for each animal, a $10 \times 10$ grid was optically superimposed on each of 20 nonoverlapping fields at $400 \times$ distributed throughout a single kidney section. (Twenty gridded fields constituted a $5 \%-10 \%$ sampling of the total cross-sectional area for the sham or intact opposite kidney and a 30\%-40\% sampling for the obstructed kidney.) The number of grid points containing blue collagen staining in the interstitium (but not perivascular or in glomeruli or tubules) was divided by the total number of points in the fields $(2,000)$ to obtain the percent fractional area of interstitial collagen deposition (23). PAS staining to accentuate the thickened, irregular basement mem- 

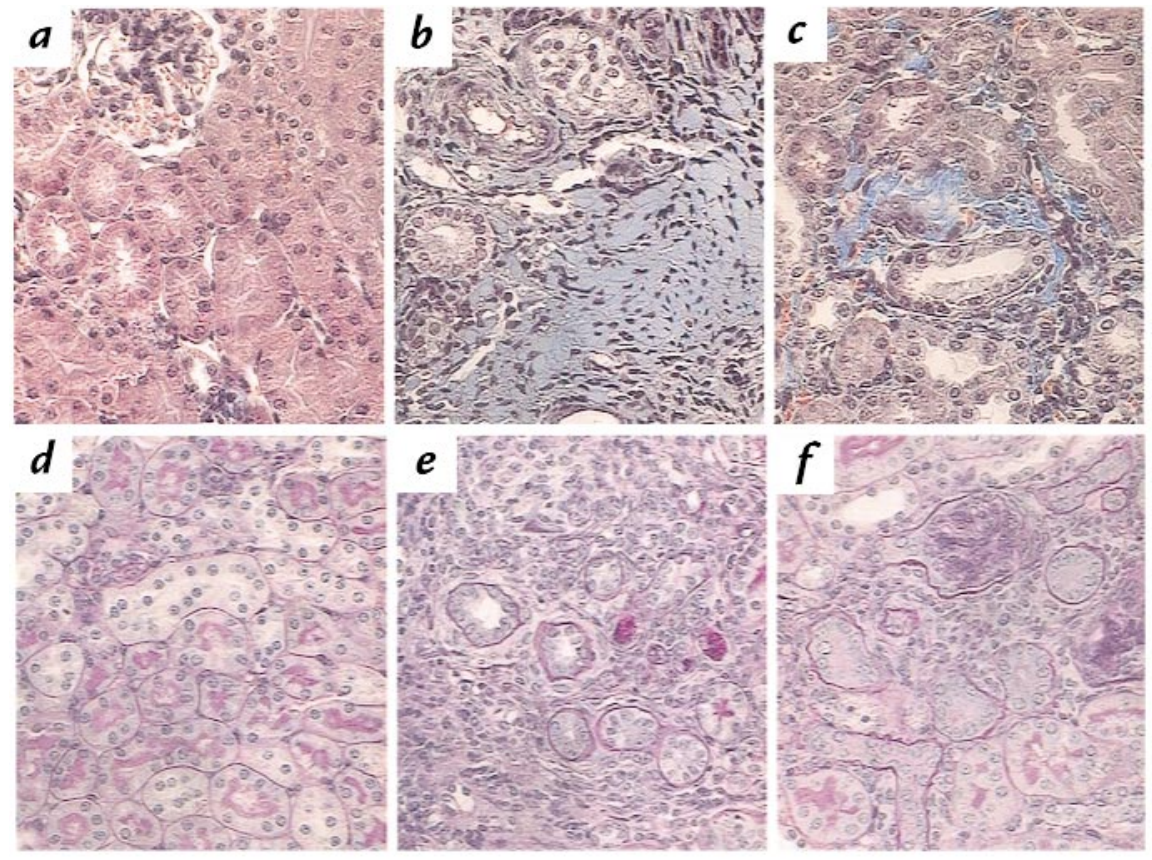

\section{Figure 2}

Renal interstitial collagen and tubular atrophy after sham operation or in chronic obstructive nephropathy during postnatal development. Renal sections from sham and obstructed mice with varying Agt expression were stained with either Masson's trichrome $(\boldsymbol{a}-\boldsymbol{c})$ to detect collagen (blue stain) or PAS $(\boldsymbol{d}-\boldsymbol{f})$ to identify the thickened, irregular tubular basement membrane characteristic of atrophy. The sham kidney of wild-type (two-copy) mice demonstrates no interstitial collagen (a) or atrophic tubules $(d)$, but UUO markedly increases both collagen deposition $(b)$ and tubular atrophy (e). Sham-operated AGT-deficient (zero-copy) mice also show evidence of collagen accumulation in the renal interstitium (c), as well as an increased proportion of atrophic renal tubules ( $f$ ). $\times 400$. PAS, periodic acid-Schiff. brane characteristic of atrophic renal tubules was performed by oxidizing renal cross-sections in $0.5 \%$ periodic acid $\times 10 \mathrm{~min}$, staining with Schiff's reagent $\times 20$ min and Gill's No. 2 hematoxylin $\times 2 \mathrm{~min}$, and then dedifferentiating with $1 \% \mathrm{HCl}$ in $70 \%$ ethanol and with $1 \%$ ammonium hydroxide $\times 5 \mathrm{~s}$ each. For each mouse, the number of atrophic tubules was quantitated under $400 \times$ magnification in the entirety of a single cross-section.

$R N A$ analysis. Total RNA was extracted from kidneys, and relative abundance of TGF- $\beta 1$ mRNA was determined by Northern blot analysis as described previously (9). Blots containing $10 \mu \mathrm{g}$ RNA in each lane were hybridized with a rat TGF- $\beta 1$ cDNA (gift of S. W. Quan, National Cancer Institute, Bethesda, Maryland, USA) and a 780-bp fragment of human glyceraldehyde-3-phosphate dehydrogenase (GAPDH; American Type Culture Collection, Manassas, Virginia, USA). The latter was used as a housekeeping gene to control for equal loading. Each blot contained RNA from the left kidney of one sham-operated mouse and left and right kidneys of UUO mice for each genotype (12 lanes), and three blots were run ( $n=3$ for each group). Hybridization signals were detected by autoradiography and quantitated by densitometric analysis. The ratio of TGF- $\beta 1$ to GAPDH mRNA was calculated for each sample.

Blood pressure measurement. Determination of blood pressure before genotype analysis was achieved noninvasively by tail-cuff sphygmomanometry using an automated, computerized, fourchannel system (Visitech Systems, Apex, North Carolina, USA) (24). Before measurement, blood pressures in animals were equilibrated $>10 \mathrm{~min}$ on the recording platform preheated to $37^{\circ} \mathrm{C}$. For each mouse, the tail-cuff pressure (in $\mathrm{mm} \mathrm{Hg}$ ) was calculated as the mean of systolic blood pressures measured in three to eight separate recording sessions during the 5 days before harvest (25-30 days old).

Statistical analysis. Data are presented as mean \pm SD and were evaluated for statistical significance using Sigmastat 2.0 (Jandel Scientific, San Rafael, California, USA). To determine the independent and combined effects of Agt genotype and UUO, comparisons between groups were performed by two-way ANOVA followed by multiple comparison testing using the Student-Newman-Keuls test. The paired $t$ test was used to evaluate differences between right and left kidney weights. The proportions of Agt genotypes within sham and UUO litters were compared with that predicted from Mendelian heritability by $\chi^{2}$ goodness-of-fit analysis; the $z$ test was performed to assess frequency differences between sham and UUO for each Agt genotype. The quantitative contribution of Agt expression to interstitial collagen deposition was assessed by simple linear regression. Statistical significance was defined as $P<0.05$.

\section{Results}

The relative survival of mice with zero, one, or two functional Agt copies after neonatal UUO $(n=87)$ or sham operation $(n=92)$ was examined because unoperated Agt $0 / 0$ mice have exhibited either increased postnatal mortality $(13)$ or normal viability $(25,26)$. Four weeks after surgery, the number of zero-copy mice was at least $48 \%$ less than that predicted from Mendelian inheritance for both sham ( $n=12$ vs. 23 expected) and UUO ( $n=7$ vs. 22 expected; both $P<0.01$ by $\chi^{2}$ analysis), consistent with an increased incidence of the Agt 0/0 genotype in mice that died prematurely (data not shown). Ureteral obstruction itself was associated with reduced viability, as the number of surviving obstructed mice per litter (6.7 \pm 1.4 , mean $\pm \mathrm{SD})$ was less than that in sham $(9.2 \pm 2.1, P=$ 0.003 by $t$ test); mortality within the first 24 h postoperatively accounted for the majority of this difference.

After 28 days of UUO, the body weight of wild-type (two-copy) mice did not differ appreciably from sham animals, nor did UUO alter body weight for any other Agt genotype (Fig. 1a). Compared with mice with one to four Agt copies, however, sham and UUO Agt 0/0 mice exhibited a similar $10 \%-37 \%$ reduction in somatic growth. When renal mass was corrected for body weight differences between groups, kidney weight in sham animals did not vary with Agt genotype (Fig. 1b). UUO in one- to fourcopy mice decreased ipsilateral kidney weight 68\%$79 \%$ compared with sham. Similarly, UUO increased contralateral renal mass $27 \%-56 \%$. In mice lacking angiotensin, obstructed kidney weight was $38 \%$ less than in sham but also 2.6-3.0 times greater than correspon- 


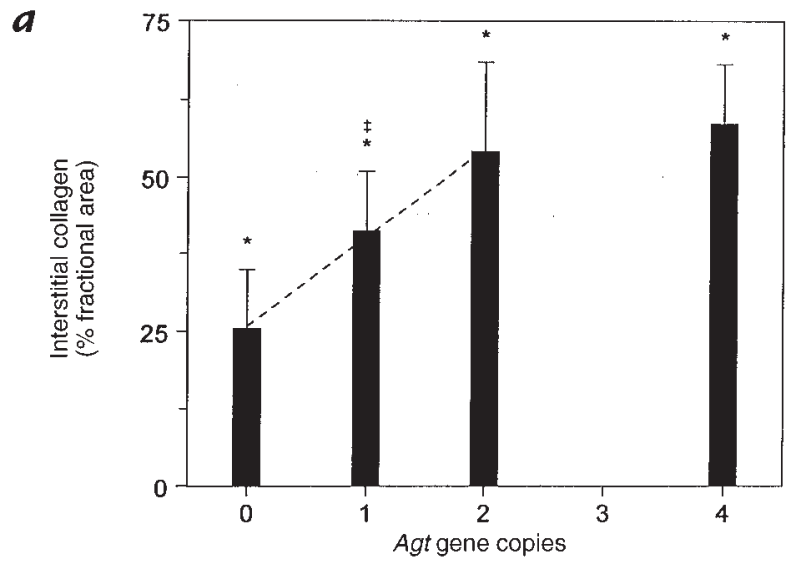

$b$

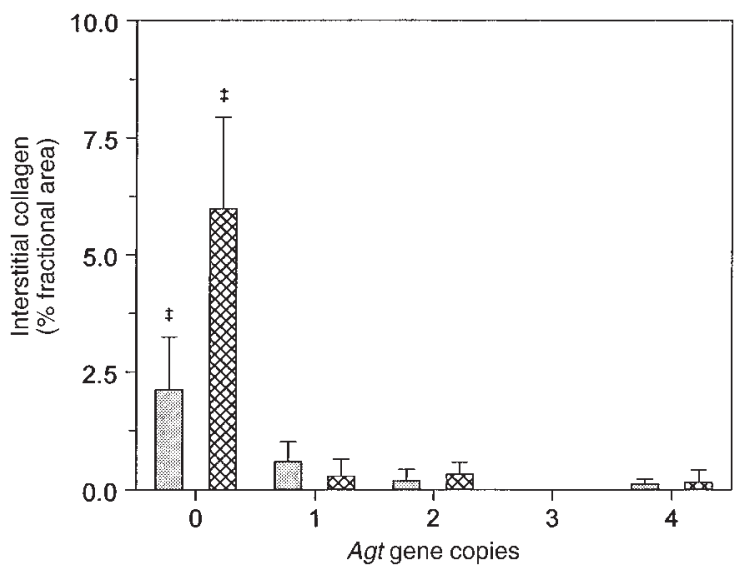

Figure 3

Dependence of UUO-induced renal interstitial collagen deposition on Agt expression. As a component of renal fibrosis, collagen accumulation in the interstitium of (a) obstructed kidneys and (b) intact opposite (crosshatched bars) and sham (gray bars) kidneys of Agt-targeted mice was localized histochemically by Masson trichrome staining and quantitated using a point-counting method by light microscopy at $400 \times$ magnification (see Methods). Data are expressed as the percent of the examined renal crosssectional area containing interstitial collagen (\% fractional area). Collagen deposition in the interstitium of obstructed and sham kidneys of wild-type (Agt 1/1) mice was $54.2 \pm 14.2 \%$ and $0.2 \pm 0.2 \%$, respectively. The ordinate scale in $b$ differs from $a$ to depict the genotypic differences in collagen staining in unobstructed kidneys. The quantitative contribution of Agt expression to collagen deposition in kidneys subjected to chronic UUO (a) was assessed by simple linear regression of individual data points from zero- to two-copy mice (dashed line; slope $=14.3 \pm 3.3 \%, R=0.72, P<0.001$ ). Data represent mean \pm SD. For each kidney type, $n=5-6,6-8,4-6$, and 3 mice with zero, one, two, or four Agt copies, respectively. ${ }^{*} P<0.05$ vs. sham. $¥ P<0.05$ vs. other Agt genotypes. $R$, multiple correlation coefficient.

ding kidneys in Agt-expressing mice (both $P<0.05$ ). By gross inspection, hydronephrotic kidneys from Agt 0/0 mice were uniformly more severely dilated and uniquely exhibited a loss of the ureteropelvic junction compared with other Agt genotypes (data not shown). In addition, UUO increased the intact opposite kidney weight of zerocopy mice by $33 \%$ (Fig. $1 b$ ), indicating that compensatory renal growth occurs in the absence of Agt expression.

Chronic obstructive uropathy is characterized by a prominent interstitial fibrosis and severe tubular atrophy (15). As an index of the fibrotic response, interstitial collagen deposition was analyzed histomor- phometrically in Masson's trichrome-stained renal cross-sections and expressed as the percent fractional area containing collagen. Collagen staining was essentially absent from the interstitium in sham and intact opposite kidneys of wild-type mice (Fig. 2a), but increased over 200-fold in the obstructed kidney to 54.2 $\pm 14.2 \%(n=6)$ (Figs. $2 b$ and $3 a)$. Decreased Agt expression in $1 / 0$ and $0 / 0$ mice resulted in significant $(P<$ $0.05)$ stepwise decrements in UUO-induced collagen (Fig. 3a). In fact, interstitial collagen deposition in the obstructed kidney exhibited a linear dependence on Agt expression in mice expressing zero to two gene copies (slope $=14.3 \pm 3.3 \%$, multiple correlation coefficient $[R]$ $=0.72, P<0.001)$ (Fig. 3a). However, fibrotic interstitial collagen in UUO was not further exacerbated in fourcopy mice. Mice without AGT also exhibited a much smaller increase in interstitial collagen in sham and intact opposite kidneys compared with other Agt genotypes (Figs. $2 c$ and $3 b$ ).

To determine whether the renal expression of the fibrogenic cytokine TGF- $\beta 1$ is regulated by Agt expression or UUO in the mouse, the relative abundance of steady-state mRNA was determined by Northern blot analysis (Fig. 4). As shown in Fig. 4a, UUO increased renal TGF- $\beta 1$ expression only in mice with functional copies of Agt, and not in zero-copy mice. There was no effect of Agt expression on TGF- $\beta 1$ expression in shamoperated or intact opposite kidneys (Fig. 4b).

Tubular atrophy in advanced renal disease is evidenced by a characteristically thickened, irregular tubular basement membrane after PAS staining of renal cross-sections. UUO increased the number of atrophic renal tubules in the obstructed kidney of two-copy mice by 94-fold relative to sham (Fig. 2, $d$ and $e$, and Fig. 5). Tubular atrophy after UUO was equivalently preponderant in other Agt genotypes (Fig. 5a). In the sham kidney, atrophic tubules were prominent only in zero-copy mice (Figs. $2 f$ and $5 b$ ), consistent with earlier observations $(13,26)$. However, the development of tubular atrophy in the unobstructed kidney of Agt $0 / 0$ mice was attenuated by $60 \% 28$ days after contralateral UUO (Fig. $5 b$ ). It should be noted that as a fraction of all tubules, tubular atrophy accounted for $>30 \%$ of tubules in obstructed kidneys compared with only $3 \%$ in kidneys of sham-operated zero-copy mice.

To assess whether the renal interstitial and/or tubular pathological changes correlated with differences in the systemic hemodynamic effect of the RAS, blood pressures of sham and obstructed animals were determined by tail-cuff sphygmomanometry. At four weeks of age, sham-operated mice with one to four Agt copies were observed to have similar systolic tail-cuff pressures, whereas Agt 0/0 mice were significantly hypotensive (Fig. 6). All animals with chronic UUO were normotensive, including zero-copy mice. Thus, during postnatal development, chronic UUO was not hypertensive in any of the mice, although it did correct the hypotension associated with the Agt 0/0 genotype. Moreover, in chronic obstructive nephropathy, the augmentation of interstitial collagen deposition by increasing Agt expression in zero- to two-copy mice occurred without blood pressure changes (Figs. 3 and 6). 


\section{Discussion}

Reduction and augmentation of endogenous RAS activity via genomic manipulation of Agt expression in mice has permitted an assessment of the quantitative contribution of Ang II to the development of obstructive nephropathy. Compared with that in wild-type mice, plasma Ang II concentration is reduced by $50 \%$ in male and $62 \%$ in female mice with one Agt copy (Kim, H.-S., and Smithies, O., unpublished observations). Thus, functional Agt expression is rate limiting in this model. Interstitial fibrosis and tubular atrophy are the principal histopathological prognostic indicators in chronic renal disease, including obstructive nephropathy $(27,28)$. UUO-induced renal interstitial collagen expression in developing wild-type mice was progressively diminished $53 \%$ by stepwise suppression of AGT production without changes in blood pressure. Renal tubular atrophy in chronically obstructed kidneys did not differ between Agt genotypes. In the absence of Agt, however, the impaired tubular integrity of hypotensive sham-operated mice was significantly improved in the intact kidney of mice with contralateral UUO and normal blood pressure. Additionally, com pensatory renal growth in response to contralateral hydronephrosis remained intact in the absence of AGT and was not accentuated by Agt overexpression.

Previous studies of the role of the RAS in the hydronephrotic kidney have relied on pharmacologic inhibition of ACE or $\mathrm{AT}_{1}$ receptors $(22,29)$. Since ACE is the same as kininase II (the primary bradykinin-degrading enzyme), these additional effects of its inhibition must also be considered: bradykinin is mitogenic and modulates TGF- $\beta 1$ stimulation of renal fibroblasts (30). Although many of the effects of Ang II are mediated by $\mathrm{AT}_{1}$ receptors, there is increasing evidence that other receptors are involved. Thus, Ang IV, through stimulation of receptors different from $\mathrm{AT}_{1}$ or $\mathrm{AT}_{2}$, induces plasminogen activator inhibitor 1 expression in renal tubular epithelial cells, thereby contributing to interstitial fibrosis (31). Thus, this Agt-targeting model provides a useful and novel tool for delineating the contribution of the RAS to kidney disease and renal growth.

Interstitial fibrosis. As demonstrated here, decreasing levels of function of the Agt gene directly cause significant reductions in interstitial collagen deposition consequent to UUO throughout early postnatal murine development (Fig. 3a). Agt overexpression, however, does not further amplify the fibrotic effect of UUO, nor does it increase interstitial collagen in unobstructed kidneys (Fig. 3) (13). In comparison, infusion of exogenous Ang II induces renal fibrosis in adult rats $(4,5)$, even at nonhypertensive doses (6). Ang II is probably maintained at higher renal levels in the rat infusion studies [three times normal (32)] than in mice with four Agt copies, in which the plasma AGT level is only about 1.5 times normal (13). Alternatively, interstitial collagen deposition after month-long UUO in wild-type animals may represent a (near) maximal level, on which further increases in Agt expression are not observed to have an effect.

As demonstrated here in Agt-targeted mice, downregulation of the RAS before and throughout chronic UUO reduces collagen protein accumulation in the renal interstitium, suggesting that Ang II influences
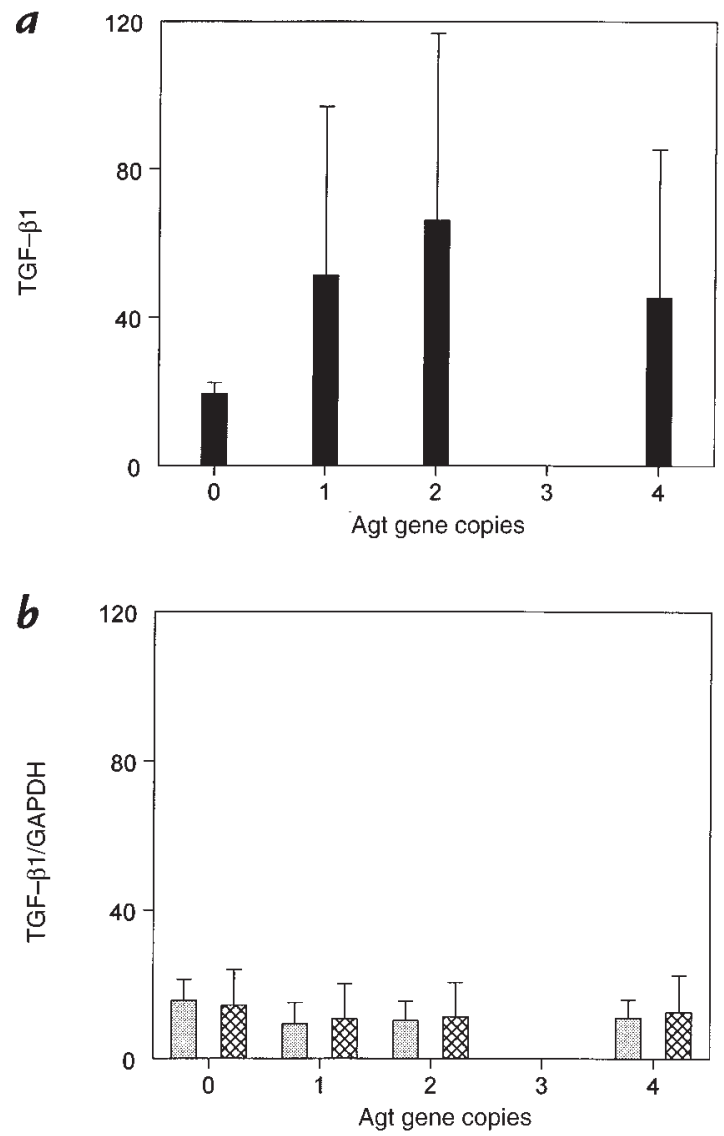

Figure 4

Northern blot densitometric ratio of TGF- $\beta 1$ to GAPDH mRNA for each group. ( $\boldsymbol{a}$ ) obstructed kidneys and $(\boldsymbol{b})$ intact opposite (cross-hatched bars) and sham (gray bars) kidneys of Agt-targeted mice. Data represent mean $\pm \mathrm{SD}$. For each kidney type, $n=3$. GAPDH, glyceraldehyde-3-phosphate dehydrogenase; $T G F$, transforming growth factor.

not only the rate but the ultimate degree of fibrosis. Clinically, therapeutic interference with Ang II action would be attempted only after disease onset. In this respect, Ishidoya et al. (29) have reported that delayed onset of ACE inhibition up to six days after renal injury still reduced disease in obstructive uropathy in adult rats. Further, Border and Noble (3) note that pharmacological attenuation of Ang II production improves renal function when applied in multiple forms of human kidney disease. Thus, angiotensin-dependent interstitial fibrosis represents a relevant target in the therapy for renal disease.

Activation of the RAS effects renal fibrotic changes potentially through multiple mechanisms. Renal injury by Ang II classically has been attributed to the systemic and renal hemodynamic consequences of the hormone's vasoconstrictor action (33). In chronic hydronephrosis in maturing animals, the influence of reduced Agt expression on renal interstitial fibrosis occurred without changes in systemic blood pressure (Fig. 6). Several candidate mediators of a fibrotic effect of Ang II in the kidney have been identified. Principal among these is TGF$\beta$. TGF- $\beta$ is upregulated in many forms of kidney injury (3), its inhibition decreases renal disease (34-36), and its 

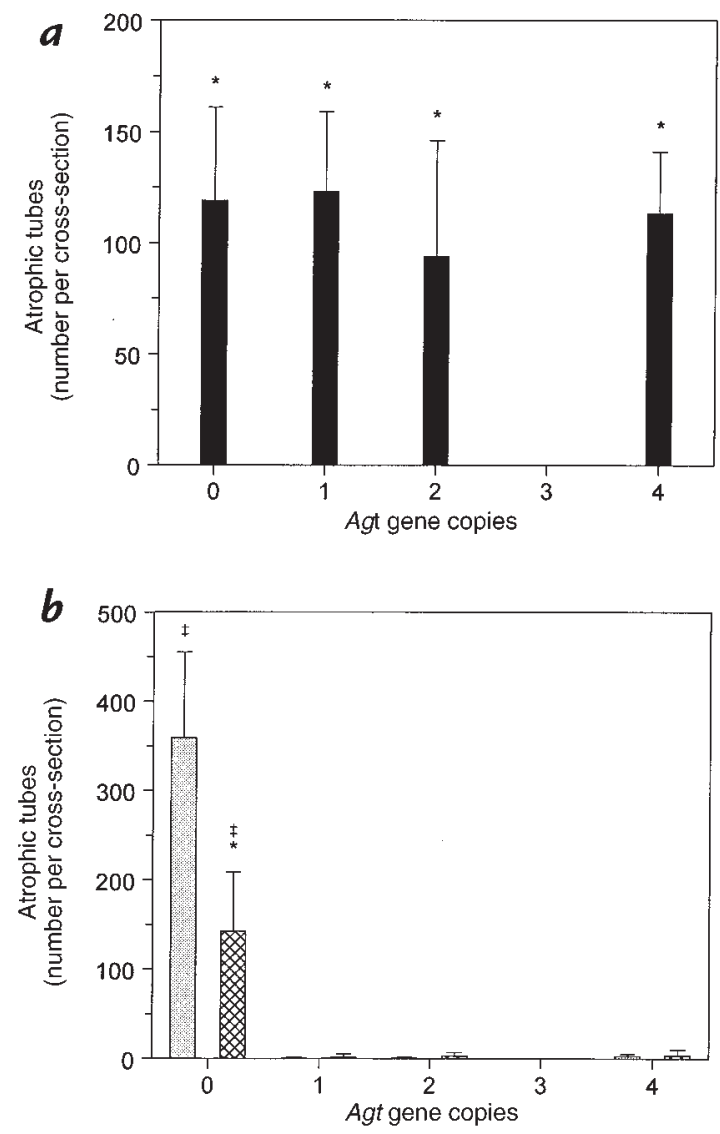

Figure 5

Effect of UUO and Agt expression on renal tubular atrophy. The number of atrophic tubules in mice with zero to four Agt copies was quantitated throughout PAS-stained cross-sections of ( $\boldsymbol{a})$ obstructed kidneys and $(\boldsymbol{b})$ intact opposite (cross-hatched bars) and sham (gray bars) kidneys. The ordinate scale in $b$ differs from $a$ because the number of atrophic tubules quantitated corresponds to $>30 \%$ of all tubules in sections of hydronephrotic kidneys, but $<3 \%$ in intact kidneys. Data represent mean \pm SD. For each kidney type, $n=5-6,6-8,4-8$, and 3 mice with zero, one, two, or four Agt copies, respectively. ${ }^{*} P<0.05$ vs. sham. $¥ P<0.05$ vs. other Agt genotypes.

overexpression produces significant renal pathology $(37,38)$. As shown in the present study, though dramatically attenuated, renal interstitial fibrosis in chronic obstructive nephropathy persists in the complete absence of Ang II, presumably the result of additional fibrotic pathways. The increase in renal TGF- $\beta 1$ expression in obstructed kidneys of mice expressing Agt, but not those with zero-copy Agt, suggests that expression of this cytokine is coupled to Ang II production. Standard antihypertensive regimens of ACE inhibitors or $\mathrm{AT}_{1}$ receptor blockers are efficacious in ameliorating renal disease of varying pathogenesis, but generally they induce only partial reduction of TGF- $\beta$ levels $(3,9)$. It has been speculated whether increasing dosages to augment Ang II inhibition would yield improved salutary effects on renal disease concomitant with suppression of TGF$\beta$ action (3). Such a strategy may have added benefit, but our results predict that maximizing Ang II blockade will not halt renal fibrosis in clinical disease.

The present work does not dismiss aldosterone as an hormonal effector of fibrotic angiotensin action in obstructive nephropathy. Recent attention to the Ang II-regulated, adrenal glomerulosa-derived mineralocorticoid reveals this hormone to contribute to disease in the remnant kidney model (39), potentially via direct cellular actions as well as through hemodynamic effects (33).

Mice lacking Agt exhibited diminished fibrosis in chronic obstructive nephropathy by $50 \%$ (Fig. $3 a$ ) but also increased spontaneous renal interstitial fibrosis in shamoperated kidneys (Fig. 3b) $(13,26)$. These observations prompt the consideration of the signaling cascade accounting for the renal morphological lesions in the absence of Ang II. The effect of AGT deficiency on interstitial collagen clearly is not synergistic with chronic UUO, because the lack of Agt both provokes renal injury during postnatal development and limits the expected extracellular matrix deposition in this model of tubulointerstitial disease. Nevertheless, using in situ hybridization techniques, Niimura and colleagues (26) found increased interstitial TGF- $\beta 1$ and tubular PDGF-B expression in areas of renal damage in mice without a functional Agt. Although we did not find an increase in TGF- $\beta 1$ expression in either intact or obstructed kidneys in zero-copy mice, this presumably reflects the reduced sensitivity of Northern analysis compared with in situ hybridization. It should be emphasized, however, that interstitial fibrosis in obstructed kidneys of zero-copy mice is 10 -fold greater than that of sham-operated kidneys (Fig. 3). Clearly, angiotensin-independent factors account for $50 \%$ of the interstitial fibrosis resulting from UUO. Increased interstitial collagen in intact kidneys of zero-copy animals also persisted, despite the correction of low blood pressure by contralateral UUO, indicating that hypotension alone did not account for the observed renal fibrotic changes.

Tubular atrophy. In progressive obstructive nephropathy, renal tubules dilate and tubular epithelial cells

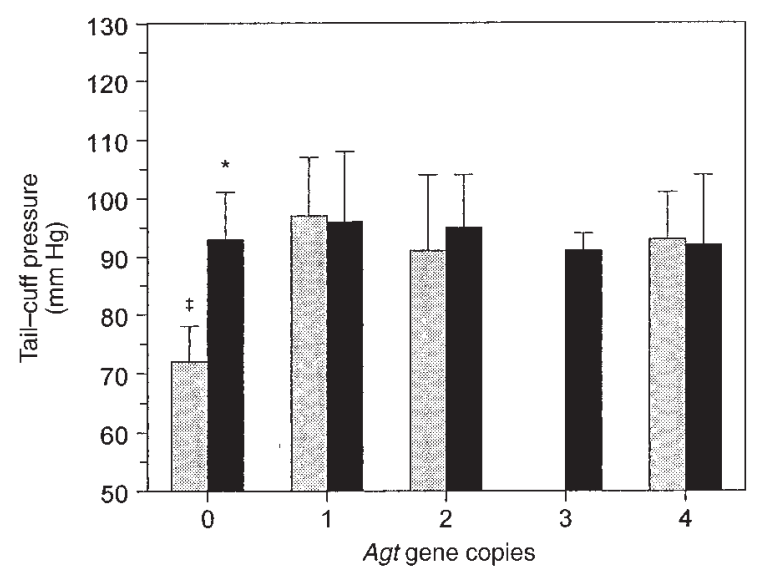

\section{Figure 6}

Blood pressure in sham and UUO mice with varying Agt expression. Tailcuff sphygmomanometry was used to assess blood pressure as a parameter of the influence of Agt copy number on systemic hemodynamics in developing mice with chronic UUO (black bars) or after sham operation (gray bars). The tail-cuff pressure in sham wild-type $(1 / 1)$ mice $(91 \pm 13 \mathrm{~mm} \mathrm{Hg})$ was similar in UUO and for all genotypes except sham Agt 0/0 (72 $\pm 6 \mathrm{~mm}$ $\mathrm{Hg})$. Data represent mean $\pm \mathrm{SD}$. For each sham or UUO value, $n=3-4$, 43-48, 11-16, 3, and 3 mice with zero, one, two, three, or four Agt copies, respectively. ${ }^{*} P<0.05$ vs. sham. ${ }^{\ddagger} P<0.05$ vs. other Agt genotypes. 
undergo apoptosis and necrosis, leading to tubular atrophy $(40,41)$. The degree of renal tubular atrophy in chronic hydronephrosis was not altered by differences in Agt expression (Fig. 5a), in contrast to collagen accumulation in the interstitium. Whether the rate of degenerative tubular changes within this time period ( 28 days) is accelerated by Ang II remains to be defined.

The absence of Agt expression did not further exacerbate the impaired renal tubular integrity observed in UUO, but occasioned tubular atrophy in sham animals as described previously $(13,26)$. Remarkably, UUO reduced the number of atrophic tubules in the contralateral intact kidney of Agt 0/0 mice, suggesting a salutary, angiotensin-independent signal promoting renal tubular integrity as a component of the "cross-talk" between the injured and intact kidneys. The relevant tubular signal may be associated with the undefined mechanism that induces compensatory growth of the same kidney in response to UUO (see below). Alternatively, regulation of systemic blood pressure may account for both the presence of renal tubular atrophy in sham-operated mice and its amelioration in intact kidneys compensating for contralateral hydronephrosis. In the absence of Agt expression, shamoperated mice with impaired tubular integrity were significantly hypotensive, but mice subjected to neonatal UUO were normotensive with improved (but not absent) tubular atrophy. Thus, renal ischemia and its correction may contribute to the differences in tubular atrophy in intact kidneys of sham and obstructed Agt-deficient mice.

Blood pressure. Blood pressure did not vary for four-weekold sham-operated mice with one to four Agt copies, although it was markedly reduced in the absence of AGT (Fig. 6). Young adult (73 to 134 days old) mouse blood pressure, in contrast, exhibits a dose dependence on Agt expression (13). In an independent Agt-targeted model, zero-, one-, and two-copy mice also displayed graded changes in blood pressure at 7 to 8 weeks of age (26). These observations may reflect a maturation of the sensitivity of the systemic vasculature to angiotensin. In comparison, during the postnatal development of the spontaneously hypertensive rat, a "prehypertensive stage" endures for several weeks, with minimal blood pressure differences compared with age-matched controls $(42,43)$. Interestingly, all Agt genotypes were normotensive after UUO in the present study. The maintenance of normotension by UUO in the zero-copy mice may relate to the production of other vasoconstrictors, such as endothelin, which are generated by the hydronephrotic kidney (44).

Renal growth and development. After chronic UUO, the increased weight of hydronephrotic kidneys of Agt-deficient mice compared with other Agt genotypes occurred in the context of more pronounced dilatation of the kidney and loss of the ureteropelvic junction, suggesting an impaired capacity of the pelvic and proximal ureteral architecture to resist the increased hydrostatic pressure with UUO in the absence of AGT. This observation is consistent with the recent report that mice deficient in signaling through the $\mathrm{AT}_{1}$ receptor lack a renal pelvis and exhibit hypoplasia of the ureteral musculature, with absence of ureteral peristalsis (45).

The issue of the influence of the RAS on compensatory renal growth has been raised, because Ang II serves as a mitogen and hypertrophic agent for proximal tubular development(46) and participates in renal development and maturational growth $(13,26,47)$. Conflicting studies have supported (48) or contested (49) an essential role for Ang II in the hypertrophy and hyperplasia compensatory to contralateral renal mass reduction. Here, contralateral kidney weight after chronic UUO in AGT-deficient mice increased to the same degree as in animals expressing various levels of Agt (Fig. 1b), demonstrating unequivocally that compensatory renal growth occurs independent of Ang II action.

In summary, neither compensatory renal growth in response to contralateral hydronephrosis nor the compromised renal tubular integrity in chronically obstructed kidneys was altered by decreased or increased RAS activity in developing mice with varied Agt expression. In intact kidneys, tubular atrophy in the absence of AGT was mitigated by contralateral UUO coincident with the correction of hypotension, implicating renal ischemia in this setting of tubular epithelial degeneration. In contrast, diminished UUO-induced renal interstitial collagen through progressive reduction of Agt expression emphasizes the quantitative contribution of RAS signaling to the fibrotic response in chronic obstructive nephropathy, possibly through regulation of TGF- $\beta 1$ expression. Dissociation of the Agt effect on fibrosis from changes in systemic blood pressure supports increasing evidence for the hemodynamic-independent role of Ang II in renal pathology. Moreover, because progressive fibrosis is a hallmark of end-stage renal disease, angiotensin-dependent fibrosis may represent a common theme in the exacerbation of kidney injury.

\section{Acknowledgments}

We acknowledge Jennifer Lindsey (University of Virginia) for provision of Agt-targeted mice maintained locally for breeding, Jennifer Wolstenholme for measurement of renal TGF- $\beta 1$ mRNA, Ariel Gomez (University of Virginia) for helpful review of the manuscript, and Robert Carey (University of Virginia) for helpful suggestions. This research was supported by the National Institutes of Health (NIH) Research Center of Excellence in Pediatric Nephrology and Urology (DK-52612), the NIH O'Brien Center of Excellence in Nephrology and Urology (DK-45179), the NIH Child Health Research Center (HD-28810), and the NIH Basic Cardiovascular Research Training grant (HL-02784).

\footnotetext{
1. Weber, K.T. 1997. Fibrosis, a common pathway to organ failure: angiotensin II and tissue repair. Semin. Nephrol. 17:467-491.

2. Noble, N.A., and Border, W.A. 1997. Angiotensin II in renal fibrosis: should TGF-beta rather than blood pressure be the therapeutic target? Semin. Nephrol. 17:455-466.

3. Border, W.A., and Noble, N.A. 1998. Interactions of transforming growth factor-beta and angiotensin II in renal fibrosis. Hypertension. 31:181-188.

4. Johnson, R.J., et al. 1992. Renal injury from angiotensin II-mediated hypertension. Hypertension. 19:464-474.

5. Kagami, S., Border, W.A., Miller, D.E., and Noble, N.A. 1994. Angiotensin II stimulates extracellular matrix protein synthesis through induction of transforming growth factor-beta expression in rat glomerular mesangial cells. J. Clin. Invest. 93:2431-2437.

6. Yoo, K.H., Thornhill, B.A., Wolstenholme, J.T., and Chevalier, R.L. 1998. Tissue-specific regulation of growth factors and clusterin by angiotensin II. Am. J. Hypertens. 11:715-722.

7. Anderson, S., Meyer, T.W., Rennke, H.G., and Brenner, B.M. 1985. Control of glomerular hypertension limits glomerular injury in rats with reduced renal mass. J. Clin. Invest. 76:612-619.

8. Kohno, M., et al. 1992. Angiotensin II stimulates endothelin-1 secretion in cultured rat mesangial cells. Kidney Int. 42:860-866.

9. Chung, K.H., Gomez, R.A., and Chevalier, R.L. 1995. Regulation of renal
} 
growth factors and clusterin by AT1 receptors during neonatal ureteral obstruction. Am. J. Physiol. 268:F1117-F1123.

10. Chevalier, R.L., Goyal, S., Wolstenholme, J.T., and Thornhill, B.A. 1998. Obstructive nephropathy in the neonatal rat is attenuated by epidermal growth factor. Kidney Int. 54:38-47.

11.Klahr, S., and Morrissey, J. 1998. Angiotensin II and gene expression in the kidney. Am. J. Kidney Dis. 31:171-176.

12. Smithies, O., and Kim, H.S. 1994. Targeted gene duplication and disruption for analyzing quantitative genetic traits in mice. Proc. Natl. Acad. Sci. USA. 91:3612-3615.

13. Kim, H.S., et al. 1995. Genetic control of blood pressure and the angiotensinogen locus. Proc. Natl. Acad. Sci. USA. 92:2735-2739.

14. Najarian, J.S., et al. 1993. Renal transplantation in the first five years of life. Kidney Int. Suppl. 43:S40-S44.

15. Moller, J.C., Skriver, E., Olsen, S., and Maunsbach, A.B. 1984. Ultrastructural analysis of human proximal tubules and cortical interstitium in chronic renal disease (hydronephrosis). Virchows Arch [A]. 402:209-237.

16. Gomez, R.A., et al. 1989. Distribution of renin mRNA and its protein in the developing kidney. Am. J. Physiol. 257:F850-F858.

17. Yosipiv, I.V., and el-Dahr, S.S. 1996. Activation of angiotensin-generating systems in the developing rat kidney. Hypertension. 27:281-286.

18. el-Dahr, S.S., et al. 1990. In situ localization of renin and its mRNA in neonatal ureteral obstruction. Am. J. Physiol. 258:F854-F862.

19. Norwood, V.F., et al. 1994. Neonatal ureteral obstruction stimulates recruitment of renin-secreting renal cortical cells. Kidney Int 45:1333-1339.

20. el-Dahr, S.S., et al. 1993. Upregulation of renin-angiotensin system and downregulation of kallikrein in obstructive nephropathy. Am. J. Physiol. 264:F874-F881.

21. Yoo, K.H., Norwood, V.F., el-Dahr, S.S., Yosipiv, I., and Chevalier, R.L. 1997. Regulation of angiotensin II AT1 and AT2 receptors in neonatal ureteral obstruction. Am. J. Physiol. 273:R503-R509.

22. Ishidoya, S., Morrissey, J., McCracken, R., Reyes, A., and Klahr, S. 1995. Angiotensin II receptor antagonist ameliorates renal tubulointerstitia fibrosis caused by unilateral ureteral obstruction. Kidney Int 47:1285-1294.

23. Sheehan, D.C., and Hrapchak, B.B. 1980. Theory and practice of histotechnology. Barelle. Columbus, OH. 188-194.

24. Krege, J.H., Hodgin, J.B., Hagaman, J.R., and Smithies, O. 1995. A noninvasive computerized tail-cuff system for measuring blood pressure in mice. Hypertension. 25:1111-1115.

25. Lindsey, J., Kim, H.S., Smithies, O., and Gomez, R.A. 1998. Angiotensinogen is necessary for postnatal kidney development. Pediatr. Res. 43:310a. (Abstr.)

26. Niimura, F., et al. 1995. Gene targeting in mice reveals a requirement for angiotensin in the development and maintenance of kidney morphology and growth factor regulation. J. Clin. Invest. 96:2947-2954.

27. Risdon, R.A., Sloper, J.C., and De, W.E. 1968. Relationship between renal function and histological changes found in renal-biopsy specimens from patients with persistent glomerular nephritis. Lancet. 2:363-366.

28. Schainuck, L.I., Striker, G.E., Cutler, R.E., and Benditt, E.P. 1970. Structural-functional correlations in renal disease. II. The correlations. Hum. Pathol. 1:631-641.

29. Ishidoya, S., Morrissey, J., McCracken, R., and Klahr, S. 1996. Delayed treatment with enalapril halts tubulointerstitial fibrosis in rats with obstructive nephropathy. Kidney Int 49:1110-1119.

30. Van Zoelen, E.J., et al. 1994. Bradykinin-induced growth inhibition of normal rat kidney (NRK) cells is paralleled by a decrease in epidermalrowth-factor receptor expression. Biochem. J. 298:335-340.

31. Gesualdo, L., et al. 1997. Angiotensin IV, inducing plasminogen activator inhibitor 1 (PAI-1) expression in proximal tubular epithelial cells, may be implicated in the pathogenesis of renal interstitial fibrosis. J. Am. Soc. Nephrol. 8:515a. (Abstr.)

32. Von Thun, A.M., Vari, R.C., el-Dahr, S.S., and Navar, L.G. 1994. Augmentation of intrarenal angiotensin II levels by chronic angiotensin II infusion. Am. J. Physiol. 266:F120-F128.

33. Ibrahim, H.N., Rosenberg, M.E., and Hostetter, T.H. 1997. Role of the renin-angiotensin-aldosterone system in the progression of renal disease: a critical review. Semin. Nephrol. 17:431-440.

34. Border, W.A., Okuda, S., Languino, L.R., Sporn, M.B., and Ruoslahti, E. 1990. Suppression of experimental glomerulonephritis by antiserum against transforming growth factor beta 1. Nature. 346:371-374

35. Border, W.A., et al. 1992. Natural inhibitor of transforming growth factor-beta protects against scarring in experimental kidney disease. Nature. 360:361-364

36. Sharma, K., Jin, Y., Guo, J., and Ziyadeh, F.N. 1996. Neutralization of TGF-beta by anti-TGF-beta antibody attenuates kidney hypertrophy and the enhanced extracellular matrix gene expression in STZ-induced diabetic mice. Diabetes. 45:522-530.

37. Isaka, Y., et al. 1993. Glomerulosclerosis induced by in vivo transfection of transforming growth factor-beta or platelet-derived growth factor gene into the rat kidney. J. Clin. Invest. 92:2597-2601.

38. Kopp, J.B., et al. 1996. Transgenic mice with increased plasma levels of TGF-beta 1 develop progressive renal disease. Lab. Invest. 74:991-1003.

39. Greene, E.L., Kren, S., and Hostetter, T.H. 1996. Role of aldosterone in the remnant kidney model in the rat. J. Clin. Invest. 98:1063-1068.

40. Eddy, A.A. 1996. Molecular insights into renal interstitial fibrosis. J. Am. Soc. Nephrol. 7:2495-2508.

41. Chevalier, R.L. 1996. Growth factors and apoptosis in neonatal ureteral obstruction. J. Am. Soc. Nephrol. 7:1098-1105.

42. Lais, L.T., Rios, L.L., Boutelle, S., DiBona, G.F., and Brody, M.J. 1977. Arterial pressure development in neonatal and young spontaneously hypertensive rats. Blood Vessels. 14:227-284.

43. Gray, S.D. 1984. Spontaneous hypertension in the neonatal rat: a review. Clin. Exp. Hypertens. 6:755-781.

44. Josephson, S., and Hemsen, A. 1994. Renal tissue endothelin in longterm complete ureteric obstruction in the young rat. Urol. Int. 53:57-61.

45. Miyazaki, Y., et al. 1998. A new role for mammalian angiotensin: adapting the kidney to extrauterine life. Pediatr. Res. 43:311a. (Abstr.)

46. Wolf, G. and Neilson, E.G. 1990. Angiotensin II induces cellular hypertrophy in cultured murine proximal tubular cells. Am. J. Physiol. 259:F768-F777.

47. Tufro-McReddie, A., Romano, L.M., Harris, J.M., Ferder, L., and Gomez, R.A. 1995. Angiotensin II regulates nephrogenesis and renal vascular development. Am. J. Physiol. 269:F110-F115.

48. Dworkin, L.D., Grosser, M., Feiner, H.D., Ullian, M., and Parker, M. 1989. Renal vascular effects of antihypertensive therapy in uninephrectomized SHR. Kidney Int. 35:790-798.

49. Valentin, J.P., Sechi, L.A., Griffin, C.A., Humphreys, M.H., and Schambelan, M. 1997. The renin-angiotensin system and compensatory renal hypertrophy in the rat. Am. J. Hypertens. 10:397-402. 